\title{
VISUAL DISORDERS WITH ANTIEPILEPTIC DRUGS
}

Visual adverse effects induced by antiepileptic drugs (AED) are reviewed by researchers at the Departments of Pediatrics, Universities of Chieti and Bologna, Italy. The majority are reversible and rare. Exceptions include vigabatrin-induced visual field loss that may be irreversible and is frequent $(20-40 \%)$, and tiagabine-induced blurred vision that is reversible but frequent $(41 \%)$. The newer AEDs cause visual disturbances more often than the older AEDs. Diplopia and/or nystagmus are the most commonly reported of AED visual disturbances, usually dose-related, and occur rarely with phenytoin, carbamazepine, oxcarbazepine, ethosuximide, felbamate, gabapentin, and lamotrigine (rotary nystagmus). Benzodiazepines may cause blurred vision, retinopathy, glaucoma, and oculomotor imbalance. Topiramate is rarely the cause of a reversible glaucoma, myopia, and ciliochoroidal detachment. Valproate may be associated with impaired visual evoked potentials, visual field defects and deficits in color vision. No adverse visual side effects are reported with levetiracetam. (Verrotti A, Manco R, Matricardi S, Franzoni E, Chiarelli F. Antiepileptic drugs and visual function. Pediatr Neurol June 2007;36:353-360). (Respond: Dr Verrotti, Department of Pediatrics, University of Chieti, Ospedale Policlinico, Via dei Vestini 5, 66100 Chieti, Italy. E-mail: averrott(a) unich.it).

COMMENT. Older AEDs, especially phenytoin and carabamazepine, are known to be a cause of reversible diplopia and nystagmus, but only when drug levels exceed the recommended therapeutic range. Of the newer AEDs, vigabatrin is associated with visual field defects that are often irreversible and related more to the duration of therapy than the dose. The possibility of subtle visual disturbances should be considered in children treated with AEDs, and especially with vigabatrin and other new agents. Ophthalmologic evaluation should be included in clinical trials of new AEDs and in long-term follow-up. The lack of robust evidence-based treatment recommendations for childhood epilepsy is criticized in the current issue of Lancet Neurology August 2007;6:663.

\section{HEADACHE DISORDERS}

\section{HEADACHE IN SICKLE CELL DISEASE}

The prevalence of frequent headache in children with sickle cell disease (SCD) compared to that of control subjects without known sickle cell trait, and the cause of the headaches were studied at the Children's Hospital of Philadelphia, PA, and Duke University Medical Center. Patients and controls were ages 6 to 21 years. Thirty two per cent of 241 children with SCD and $27 \%$ of 141 black control subjects reported having headaches at least weekly, a prevalence not significantly different $(\mathrm{P}=0.27)$. Mean age of those with frequent headache was $14.2+/-4.3$ years for SCD patients and $15.9+/-3.2$ for controls $(\mathrm{P}=0.03)$. In children $<13$ years old, prevalence of frequent headache was $24 \%$ for SCD and $9.7 \%$ for controls ( $\mathrm{P}=0.013)$. In both groups, headache was more common with increasing age $(\mathrm{P}<0.001)$. Prevalence was similar in various SCD genotypes (SSD-SS, SCD-SC, SCD-SBthalassemia). Headaches meeting IHS criteria for migraine occurred in $22.1 \%$ of patients with SCD and in $21.1 \%$ controls. Children with frequent headache were more likely to report 
specific SCD-related factors such as frequent vaso-occlusive pain episodes (VOE) that occurred concurrently with headache: obstructive sleep apnea (OSA) that may cause headaches; and cerebral vessel stenosis and vasculopathy detected by MR angiography. Silent cerebral infarction or stroke was not associated with headache. Abnormal transcranial Doppler (TCD) together with abnormal MRA were associated with frequent headache. Screening with TCD may be helpful in the evaluation of frequent headache and the risk of stroke. (Niebanck AE, Pollock AN, Smith-Whitley K et al. Headache in children with sickle cell disease: prevalence and associated factors. J Pediatr July 2007;151:67-72). (Reprints: Janet L Kwiatkowski MD, The Children's Hospital of Philadelphia, 3535 Market Street, Room 1571, Philadelphia, PA 19104).

COMMENT. The overall prevalence of headaches in children with SCD is not greater than that in the general population. Younger children with SCD have headaches more frequently than control subjects without sickle cell trait. SCD-specific factors such as vasoocclusive episodes and cerebral vessel stenosis may contribute to the headaches.

\section{MIGRAINE IN ADOLESCENTS AND SOCIOECONOMIC STATUS}

The epidemiology of migraine, social causation, and patterns of medical treatment among adolescent migraineurs were studied in 120,000 households in various regions of the United States and reported from Albert Einstein College of Medicine, Bronx, NY. A total of 32,015 adolescents, ages 12 to 19 years, was identified by questionnaire survey. The response rate was $58.4 \%$, and was similar in boys and girls and across age groups. The overall 1-year period prevalence of migraine was $6.3 \% ; 5.0 \%$ for boys and $7.7 \%$ for girls. Prevalence peaked at 17 years; $5.6 \%$ at 17 vs $4.6 \%$ at age 12 (in boys, $4.1 \%$ vs $3.4 \%$, and in girls, $7.8 \%$ vs $3.2 \%$, respectively). This was attributed to the stress of senior year at high school. Prevalence was significantly higher in white than African American subjects. Migraine prevalence was significantly related to low household income, especially in families without a family history of migraine. Overall, $61 \%$ subjects had from one to four severe headaches per month. Acute treatment was over-the-counter drugs only in $59.3 \%$, prescription medication in $16.5 \%$, and both were used in $22.1 \%$, most commonly in girls, whites, and older subjects. Migraine preventive treatment was never used in $63.7 \%$, and was currently in use in $16.9 \%$. Environmental factors related to low income and migraine prevalence, including nutrition, were suspected. (Bigal ME, Lipton RB, Winner P et al. Migraine in adolescents: Association with socioeconomic status and family history. Neurology July 3 2007;69:16-25). (Reprints: Dr Marcelo E Bigal, Albert Einstein College of Medicine, 1300 Morris Park Ave, Rousso Bldg, Room 330, Bronx, NY 10461).

COMMENT. Adolescents with migraine have one to four severe attacks per month; most treat with OTC only, $16.5 \%$ use prescription medicines, and only $10 \%$ use preventive treatment. An overall prevalence of migraine of $6.3 \%$ was related to low socioeconomic status and household income, suggesting social causation and the need to investigate environmental factors such as nutrition and stressors. A peak in prevalence at 17 years was thought to reflect the stress of senior year at high school and application to college. 